\title{
Polymer/Layered Compound Nanocomposites: a Way to Improve Fire Safety
}

\section{of Polymeric Materials}

YUAN HU*, XIAODONG QIAN, LEI SONG, HONGDIAN LU.

State Key Laboratory of Fire Science, University of Science and Technology of China, Hefei, Anhui 230026, PR China.

* Corresponding author email: yuanhu@ustc.edu.cn

\begin{abstract}
The review on the fire safety of polymer/layered compounds nanocomposites are summarized and discussed. Emphasis on flammable performance of polymer nanocomposites containing layered compounds, mainly montmorillonite, layered double hydroxides, layered metal phosphate, layered carbon materials and the compounds combined with conventional flame retardant additives, are also introduced based on the open literatures. Derived from those research, the combination of multiple methods and technologies including the catalyzing effect metal ion and the barrier effect of the layered compounds, are predicted to have a high probability to enhance char formation, restrain the release of combustible gases and improve the flame retardancy of polymeric materials.
\end{abstract}

Keywords: polymer nanocomposites; layered compounds; flame retardancy.

\section{Introduction}

\subsection{The flammability and fire hazard of polymeric materials}

With the development of polymer industry, the polymer materials are widely used in various areas. However, the severe environmental conditions of the polymeric materials are becoming severe.[1,2,3] For most polymeric materials, their disadvantage in intrinsic flammability makes them easily ignited by external heat. Thus, smoke and even toxic gases released during the combustion are the main cause of the loss of life and property. Generally, many serious fire accidents caused by polymeric materials have been reported widely each year and the losses of life and property have become the concern among the quality inspection authority, manufacturers and consumers. As a result, the research of flame retardants to reduce flammability of the polymers and decrease the release of toxic gas toxic during the combustion is the concern of recent scientific work and becomes an important part of the application of polymeric materials. [4,5,6,7]

\subsection{The flame retardancy of polymeric nanocomposites}

As for the flame retardancy of the polymeric materials, the early studies only focused on the fire risk scenario and the flame-retardant level. Currently, the halogenated compounds based flame retardants are still the most efficient gas-phase flame retardants and the mechanisms are based on suppression of ignition and retarding the spread of the flame. However, those flame retardants are criticized by environmental groups due to their potential hazards to the environment. Moreover, more and more people have realized the more serious problem derived from those fire hazards of polymeric materials, such as the formation of smoke and toxic gaseous products during the combustion.[8,9,10] Therefore, it is necessary to develop novel environment-friendly and efficient flame retardants to balance the flame retardant performance and the environment problems. In the past few decades, nanotechnology is one of the most popular technologies for current research and development in basically all technical disciplines and academic and industrial.[11,12,13] Moreover, many researchers have paid extensive attention to polymeric nanocomposites with the addition of the nano-sized inorganic fillers. In those fields, many diverse research directions including the nanocomposites reinforcement, the barrier properties, the electro-optical properties, the flame-retardant properties, the biological properties are the focus.[14-19] Over the past few decades, Gilman, Wilkie, Kashiwagi, Bourbigot and Camino groups, etc., have explored polymeric based nanocomposites with improved flame retardant properties by incorporating a small amount of nano-additive, such as montmorillonite (MMT), carbon nanotubes (CNT), graphene, layered double hydroxides (LDHs) and polyhedral oligomeric silsesquioxane (POSS) into the polymer matrix.[20,21,22,23] In order to improve the mechanical properties, the flame-retardant properties and reduce the cost of polymeric materials, the nano-fillers are usually incorporated into the polymer matrix during the workpiece and polymerization processes.

\subsection{The flame retardancy of polymer/nano-fillers nanocomposites}

With the development of nanotechnology, more and more groups from governmental departments, research institution and universities have participated in the research on flame retardancy of polymeric materials by incorporating the nano-fillers into the polymeric matrix.[20,21,22,23] The properties of the nanocomposites, including mechanical, thermal, flame retardancy and combustion properties, were usually investigated by X-ray diffraction (XRD), transmission electron 
microscopy (TEM), thermogravimetric analyses (TGA), Fourier transfer infrared (FTIR) spectra, Limiting Oxygen Index (LOI) and cone calorimetry. Moreover, the structure-property, pyrolysis products and combustion mechanisms of these nanocomposites are usually investigated by the real time IR line scan (RTIR), thermogravimetric analysis-infrared spectrometry (TG-IR), X-ray photoelectron spectroscopy (XPS) or scanning electron microscope (SEM). Owing to the well dispersion of nano-fillers in the polymeric matrixs, those nanocomposites exhibited greater mechanical, thermal or combustion properties when compared with the pure polymer or the conventional polymer composites.[24,25] Moreover, one can distinguish the three commonly used nano-fillers, depending on how many dimensions of the dispersed particles are in the nanometer range: one dimension, two dimensions and three dimensions. Among those nano-fillers, the two dimensional nano-fillers attracts more attention due to its barrier effect, which could inhibit the release of combustible gases and improve the thermal stability of the nanocomposites. Thus, in this review, developments in the areas of flame retardant nanocomposites using the layered compounds are introduced and discussed.

\subsection{The methods and parameters used to quantify fire performance of polymer}

Heat release rate is the important variable that determines the contribution to compartment fire hazard from materials. Thus, the flammability of the samples is usually characterized by the cone calorimeter according to the ISO 5660 . Usually, all the samples were irradiated horizontally at the heat flux of $35 \mathrm{or} 55 \mathrm{~kW} \cdot \mathrm{m}^{-2}$. Each specimen, with the dimensions of $100 \times 100 \times 3 \mathrm{~mm}^{3}$, was mounted on aluminum foil and placed on a holder filled with the mineral fiber blanket, so that only the upper face was exposed to the radiant heater. The following are the reasons: first, it is directly related to mass loss rate. The toxic fire hazard of a material is a function of the release rate of toxic gases, which is the product of total mass loss rate and yield of these gases. Second, the heat released by a material burning in a compartment results in a temperature rise of the hot layer gases and compartment walls and ceiling. With compartment fire hazard assessment as the primary application, there is a need for high quality heat release rate data, and, consequently, for devices and methods to measure the heat release accurately.

The limiting oxygen index (LOI) is the minimum concentration of oxygen, expressed as a percentage that will support combustion of polymer. It is measured by passing a mixture of oxygen and nitrogen over a burning specimen, and reducing the oxygen level until a critical level is reached. Moreover, the LOI values for different plastics are determined by standardized tests, such as the ISO 4589 and ASTM D2863. According to ASTMD2863, the specimens used for LOI were usually of dimensions $100 \times 6.5 \times 3 \mathrm{~mm}^{3}$. The specimens used for vertical burning test is $100 \times 13 \times 3 \mathrm{~mm}^{3}$.

UL 94, the standard for safety of flammability of polymer for parts in devices and appliances testing is a plastics flammability standard released by Underwriters Laboratories of the USA. UL-94 vertical burning tests were performed with plastic samples of dimensions $130 \times 13 \times 3 \mathrm{~mm}^{3}$, suspended vertically above a cotton patch. The classifications are defined according to the American National Standard UL-94: (1) V-2 burning stops within 30 seconds on a vertical specimen; drips of flaming particles are allowed; (2) V-1: burning stops within 30 seconds on a vertical specimen; drips of particles allowed as long as they are not inflamed; (3) V-0: burning stops within 10 seconds on a vertical specimen; drips of particles allowed as long as they are not inflamed.
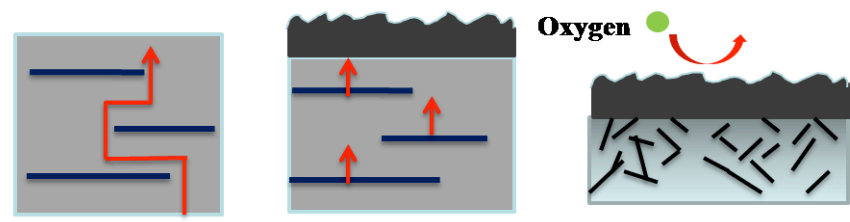

\section{Barrier effect Migration effect Catalyzing charring}

Figure 1 The fore mechanism of the layered compounds in the flame retardancy of polymer.

Based on the measurement, the flame retardant properties of the materials can be generally listed as the followings: Peak Heat Release Rate (pHRR), Total Heat Release (THR), Time to Ignition (TTI), UL-94 and LOI. As for the flame retardant mechanism, the mechanism of polymer/layered compound nanocomposites can be generally divided into three key points (Figure 1): the barrier effect, the migration effect, and the catalyzing charring effect. Generally, the pure polymer has high pHRR values, however, when the key points play their roles in the condensed phase, the nanocomposites exhibited reduced pHRR, which are due to the previous three effects. Although the layered compounds could reduce the pHRR of the nanocomposites, the layered compounds contribute little to the reducing of THR. However, when the traditional flame retardants are combined with the layered compounds, the nanocomposites usually exhibit high flame retardant efficiency especially the THR. 


\section{Polymeric nanocomposites}

2.1 Layered inorganic materials including montmorillonite, layered double hydroxides, layered metal phosphate and graphene

\subsubsection{Montmorillonite}

Montmorillonite (MMT), known as clay, consists of platelets with an inner octahedral layer sandwiched between the two silicates, as shown in Figure 2.[26] The tetrahedral sheets in which silicon is surrounded by four oxygen atoms and the octahedral sheets in which the metal like aluminum are surrounded by eight oxygen atoms, are their basic building blocks. As a result, the tetrahedral sheet is fused with an octahedral sheet in the 1:1 layered structures and the oxygen atoms are shared by the octahedral sheet.[26.27] Moreover, the octahedral layers are thought as an aluminum oxide sheet where the aluminum atoms can be replaced with many other ions. Isomorphic substitution within the layers (for example, $\mathrm{Al}^{3+}$ replaced by $\mathrm{Mg}^{2+} \mathrm{or} \mathrm{by}^{3+}$, or $\mathrm{Mg}^{2+}$ replaced by $\mathrm{Li}^{+}$) generates negative charges that are counterbalanced by alkali and alkaline earth cations situated inside the interlayers. Moreover, according to the sources, various structural compositions and sizes of montmorillonite can be varied. [28,29] However, the impurities of the limit the use of natural montmorillonites in some applications. As a result, one way to avoid these disadvantages is to synthesize the layered montmorillonite, which contains fewer drawbacks. This allows the control of the chemical composition by fine tuning the preparation methods. This method therefore provides a way to introduce some transition metal ions such as iron ion into the crystal lattice of montmorillonite, which is expected to endow the polymer nanocomposites with interesting properties.

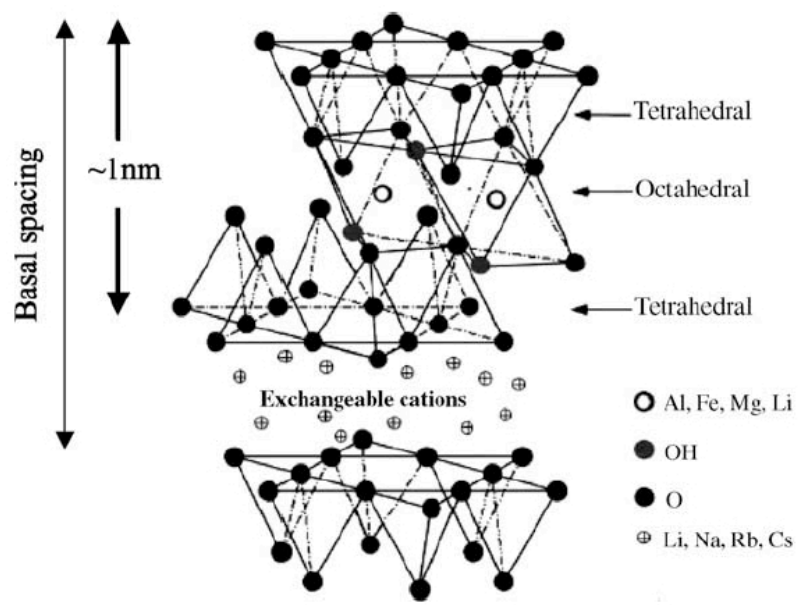

Figure 2. Structure of 2:1 phyllosilicates. Reproduced from by permission of Elsevier Science Ltd, UK.

\subsubsection{Layered double hydroxides}

LDHs, known as anionic clays, are layered inorganic materials with positive charge on the clay-like sheets compensated by interlayer anions. Moreover, the LDHs can be obtained as naturally occurring minerals or as synthetic materials and the layered double hydroxides can be considered complementary to aluminosilicate clays. Generally, the LDHs can be intercalated with the neutral guest molecules or be exchanged inorganic and organic ions for interlayer ions, which has attracted considerable attention.[30,31,32,33] Through the incorporation of the nano-layers into the polymer, the nanocomposites may have desirable physical and chemical properties. Moreover, the incorporation of organic molecules into inorganic layered hosts in general could improve the dispersion of the layered compounds and has been extensively investigated in the previous work.

\subsubsection{Layered metal phosphate}

It's known that the oxygen atom belonging to $\mathrm{PO}_{4}{ }^{3-}$ groups can be shared in both different ways and different numbers. From those combinations, amorphous precipitates or crystalline solids with fibrous, layered or three-dimensional morphology can be obtained, depending on the experimental conditions. The layered metal phosphates, such as $\mathrm{HNiPO}_{4} \cdot \mathrm{H}_{2} \mathrm{O}, \mathrm{Mn}\left(\mathrm{H}_{2} \mathrm{PO}\right)_{2} \cdot \mathrm{H}_{2} \mathrm{O}$, $\mathrm{Zn}\left(\mathrm{H}_{2} \mathrm{PO}_{2}\right)_{2} \cdot \mathrm{H}_{2} \mathrm{O}, \mathrm{Zr}\left(\mathrm{HPO}_{4}\right)_{2} \cdot \mathrm{H}_{2} \mathrm{O}$, $\alpha$-titanium phosphate, layered aluminophosphate and so on, are considered as important inorganic compounds. The crystalline of those layered metal phosphate with a solid acid catalyst function and layered structure, are intercalation compounds with good thermal stability.[34,35,36] Moreover, the layered metal phosphate has shown promise as an alternative nanoplatelet to montmorillonite for developing the primary mechanisms of polymer/layered inorganic nanocomposites, since the layered metal phosphate usually possess high ion-exchange capacity, narrow particle size distribution and controllable aspect ratio. 


\subsubsection{Layered carbon materials including expanded graphite, graphite oxide and graphene}

Expanded graphite (EG), which is explored as fillers for the polymer matrix, is produced by heating sulphuric acid-intercalated graphite. Expanded graphite (EG) is composed of many graphene sheets, which are held together by the van der Waals forces and $\pi-\pi$ interactions. This usually lead to the fact that most of the graphene sheets in the stacks could not effectively interact with the polymer matrix when the EG are incorporated into the polymeric matrix. [37,38] However, the EG can be turned into thinner graphitic nanoplatelets (GNPs) through the ultrasonic method or strong shearing method.

One other carbon nano-additive of interest is graphite oxide (GO), which are the focus of many researchers. GO is obtained when bulk graphite is exposed to strong oxidizers such as sulfuric acid, potassium chlorate, nitric acid or potassium permanganate.[39,40,41] The introduction of the oxygen-containing functional groups, including hydroxyl and epoxy groups facilitates the easy exfoliation of the graphene layers and imparts the GO the characters of water solubility. The enhanced processability of GO are benefit for the GO to be well dispersed in the polymer matrix.

Graphene, a sp ${ }^{2}$-bonded one atom-thick two-dimensional carbon layers, has attracted considerable attention in recent years due to its unique properties such as high intrinsic carrier mobility, high mechanical strength, tunable band gap and elasticity. Theoretically, the graphene has been studied for many years, and is widely used for describing properties of various carbon-based materials.[42,43,44] In the previous research, it's reported that the applications of carbon reinforcing fillers, such as carbon nanotubes (CNT), expanded graphite (EG) and graphene could reduce the flammability of polymeric materials.[45,46,47] However, to enhance the flame retardancy of polymeric materials, graphene is highly desirable due to its layered structures, carbon characteristics, large aspect ratio and low cost. Moreover, graphene is carbon materials which shows good compatibility with the formed amorphous carbon and could act as additional char layers during the combustion. Thus, the single layered graphene, which could be dispersed well in the polymer matrix, are the ideal layered flame retardants.

\subsection{Combination of layered compounds based nanocomposites}

\subsubsection{MMT-Flame retardants}

Polymer-layered silicate nanocomposites, which are the subject of the present contribution, are prepared by incorporating the layered silicate materials in a polymer matrix. However, due to hydrogen-bond interaction and the face-to-face stacking, the layered compounds could not usually be well dispersed in most polymers matrix. The most convenience methods are the melt compounding because this method is generally considered more economical and are close to the industrial application of MMT. While far from a completely accurate or descriptive nomenclature, three types of morphology are generally formed: immiscible, intercalated and exfoliated. Generally, the complete exfoliation of the clay is the ideal goal in the formation of nanocomposites. The bad dispersion of the silicate into discrete monolayers is further hindered by the further application of the clay in the industrial production. Therefore, the modifications of layered silicates with organic compound are necessary before the preparation of clay based nanocomposites.

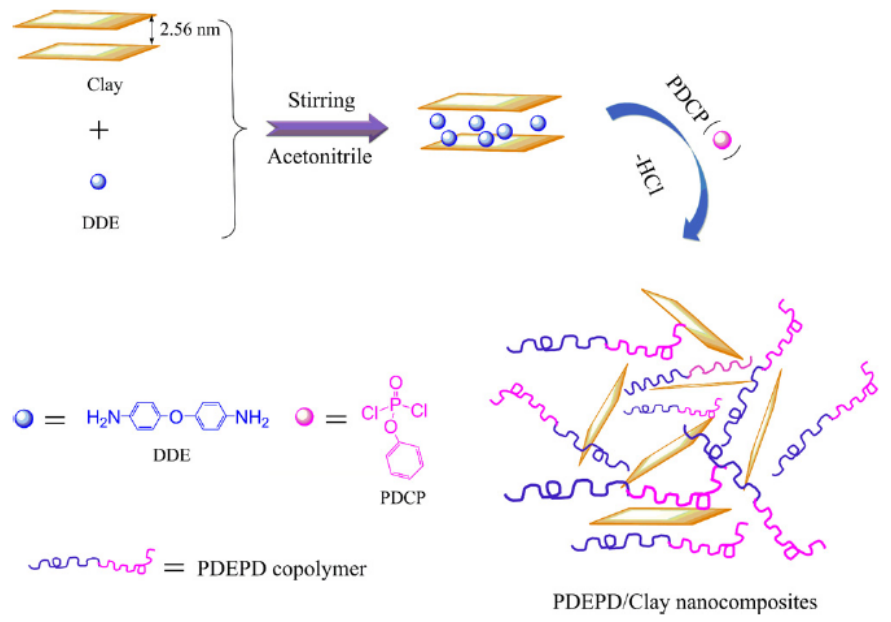

Figure 3. The schematic synthesis procedure of PDEPD/clay nanocomposites.

Among the organic modifiers, the double amino materials are the ideal modifiers to increase the layer spacing of MMT, which are also benefit for the well dispersion of MMT in the polymeric matrix. As a result, our groups present a one-step preparation of novel nanocomposites consisting of a polymeric flame retardant (PDEPD) and clays, as shown in Figure 3.[48] The clay was exfoliated or intercalated in the PDEPD and then the flame retardants containing exfoliated or intercalated clays were incorporated into the polymeric matrix, which are benefit for the dispersion of clay in the polymer matrix. As for the 
nanocomposites, the flame retardants (PDEPD/clay nanocomposites) were incorporated into polystyrene (PS) and polyurethane (PU) matrix. The flame retardancy of the composites was evidently improved, as shown in the Figure 4. Those improved flame retardant efficiency are due to the fact that the clay layers were almost exfoliated in polystyrene matrix. Generally, the well dispersions of the clay are due to the previous dispersion of the clay in the flame retardants and the combination of both flame retardants and clays could improve the flame retardant efficiency. Thus, the PDEPD/clay nanocomposites can be used as novel halogen-free flame retardant agents in many general polymer materials and the clay/flame retardants create a new preparation method of novel organic/inorganic flame retardants.
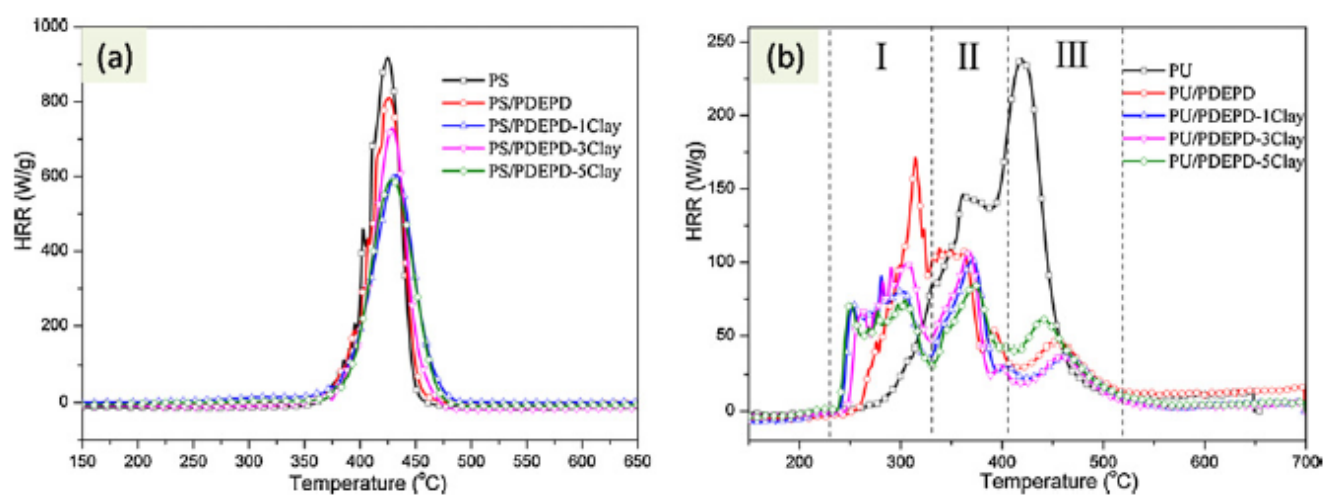

Figure 4. The heat release rate curves of (a) PS, PS composites, and (b) PU, PU composites, evaluated

by microscale combustion calorimeter (MCC)

Intumescent flame retardants (IFRs) are flame retardants which form the intumescent char layers to retard the transfer of oxygen and heat during the combustion of the composites. Generally, the clay could not only improve the flame retardant efficiency of phosphorus based flame retardant, but also could improve the flame retardant efficiency of IFR system. The combination of IFR with clay has exhibited better effect on enhancing the char properties and imparting the materials with better flame retardancy. In our previous work, flame-retarded EVA/IFR/OMMT nanocomposites crosslinked by the electron beam irradiation was investigated.[49] 1wt\% OMMT in IFR/EVA nanocomposites could increase the LOI value from $30.5 \%$ to $33.5 \%$, and UL-94 rating from V-2 to V-0, exhibiting improved flame retardant efficiency. The improved flame retardant properties of the nanocomposites are due to the followings two factors: 1.The reinforcement effect of the clays in the intumescent char layers; 2. the barrier effect of the layered structures of clays. The well dispersion of clays in the nanocomposites may result in better barrier effects, as shown in Figure 5.
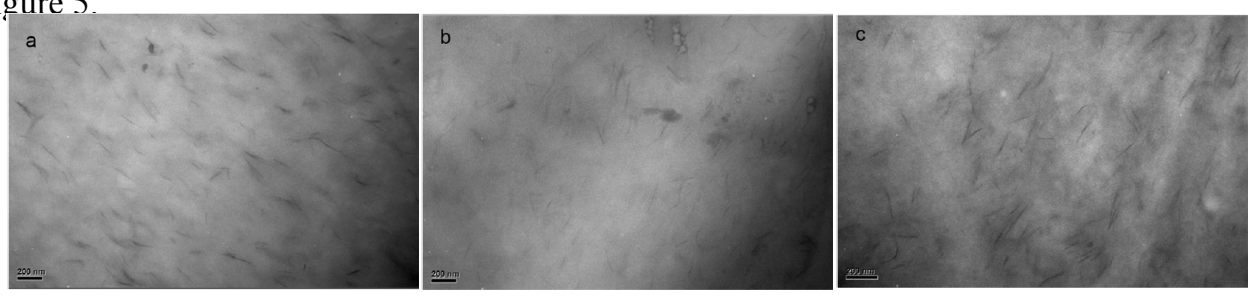

Figure 5. TEM micrographs of EVA/IFR/OMMT nanocomposites with different irradia-tion doses: (a) $0 \mathrm{kGy}$, (b) $160 \mathrm{kGy}$, and (c) $300 \mathrm{kGy}$.

Generally, the metal ions such ferric ion or nickel ion has some positive effect on flame retardancy. Thus, in order to improve the flame retardant efficiency of the polymeric materials, the ion-exchanged layered silicates, which contain flame-retardant ions such as ferric ion are usually employed. Improved thermal stability and flame retardancy for several typical polymer nanocomposites with the synthesized Fe-OMT (organically modified Fe-MMT, where $\mathrm{Al}^{3+}$ inside the crystal lattice of montmorillonite is replaced by $\mathrm{Fe}^{3+}$ have been adopted.[50,51] Generally, the improvements of the flame retardant properties are due to both the strong interactions between the silicate layers and the polymeric chains, and the presence of $\mathrm{Fe}^{3+}$ could catalyze the formation of char layers, which has been confirmed by our previous work. During thermal degradation, $\mathrm{Fe}^{3+}$ could promote the vulcanization of polymers and increase the char formation.

Generally, the addition of small amount of clay into flame retardant polymer matrix can reduce the pHRR greatly as compared with pure polymer or FRs/polymer system, irrespective the intercalation or exfoliation of the clay. However, the THR of the nanocomposites is not usually decreased significantly as compared with pHRR. Furthermore, the ignition of the nanocomposites always occurs earlier than pure polymer, [52, 53] This phenomenon can be rationally interpreted as follows: (1) organic surfactants in modified clay decompose earlier than polymer and this result in shorter ignition time; (2) the polymer matrix as well as the 
organic surfactants in the clay will be burnt out which result in the less decreased THR value; (3) the potential catalytic effect promoting the degradation of the polymeric matrix and (4) the higher viscosity of the melt inducing accumulation of heat. The decreased pHRR indicated that the reduction of burnable volatiles is due to the delayed degradation of polymer matrix. Moreover, the flame retardancy was improved due to the fact that the heat release was spread over a much longer period of time.

\subsubsection{Layered double hydroxides-Flame retardants}

Layered double hydroxides (LDHs) are layered inorganic materials with positive charge on the clay-like sheets and they are an emerging class of layered compounds for preparing polymer/layered inorganic nanocomposites. A series of LDHs have been synthesized and incorporated into the polymeric matrix by melt blending or in situ polymerization. [54,55,56] LDHs are interesting anionic clays which may play an important part in the development of fire retardant materials because the addition of LDH has been usually shown to enhance thermal stability as well as the fire safety. However, little work has studied in UV-curable acrylate resin/LDH hybrid materials. In our previous work, the novel UV-cured polymer/LDH coatings were prepared and characterized. [57] The combustion and thermal behaviors of the UV-cured films were investigated and the flame retardant properties of the nanocomposites were greatly improved, as shown in Figure 6. Moreover, the evolved products of the coating were characterized by thermogravimetric analysis-infrared spectrometry (TGA-IR) and it was found that the LDH also possesses the ability of smoke suppression. Generally, the addition of LDH was found to reinforce both the flame retardancy and physical properties of the UV-curable films.
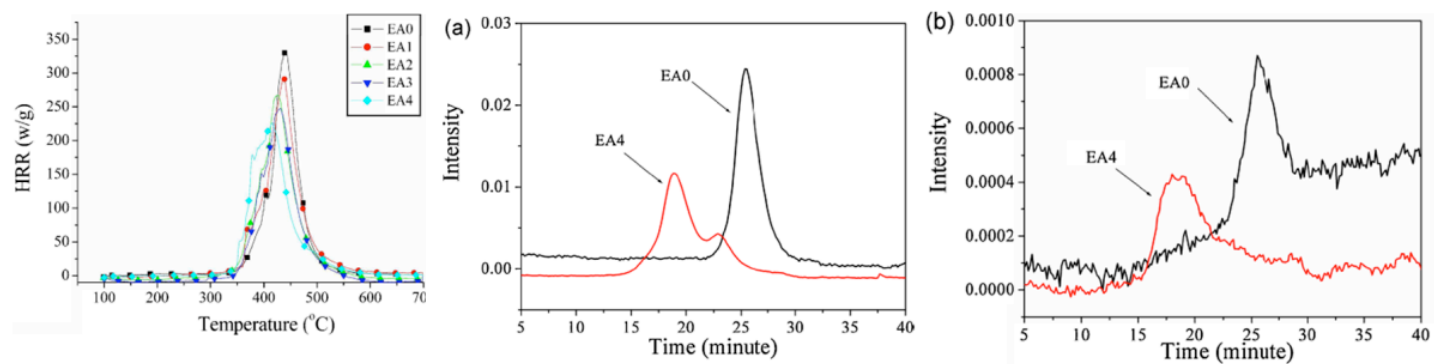

Figure 6. (a) The MCC results of the nanocomposites, (b) and carbon monoxide (CO) (c) as a function of time.

Similar to montmorillonite, the combination of $\mathrm{LDH}$ with other flame retardants, will improve flame retardancy of polymer materials. The LDH containing different divalent cations had various effects on improving flame retardant efficiency of polymer/FRs system. In our previous work, Shan explore the effects of LDH-SDS and hexaphenoxy cyclotriphosphazene (HPCP) on the thermal behavior and flammability of PLA.[58] The hydrotalcite-like layered double hydroxides with dodecylbenzenesulfonate anions (Ni-Fe, Ni-Al, and Ni-Cr LDH-SDS, where SDS denotes sodium dodecyl sulfate) were prepared by the coprecipitation method. This work studied the thermal stability and flame retardancy of PLA/HPCP/LDH-SDS composites. The thermal analysis and char residue analysis of PLA/HPCP/LDH-SDS composites suggested that Ni-Fe, Ni-Al, and Ni-Cr LDH materials play different roles in improving the thermal stability and flame retardancy of PLA composites (Figure 7). However, the incorporation of LDH usually results in the early degradation of the nanocomposites, which restrict the further application of the polymeric materials.
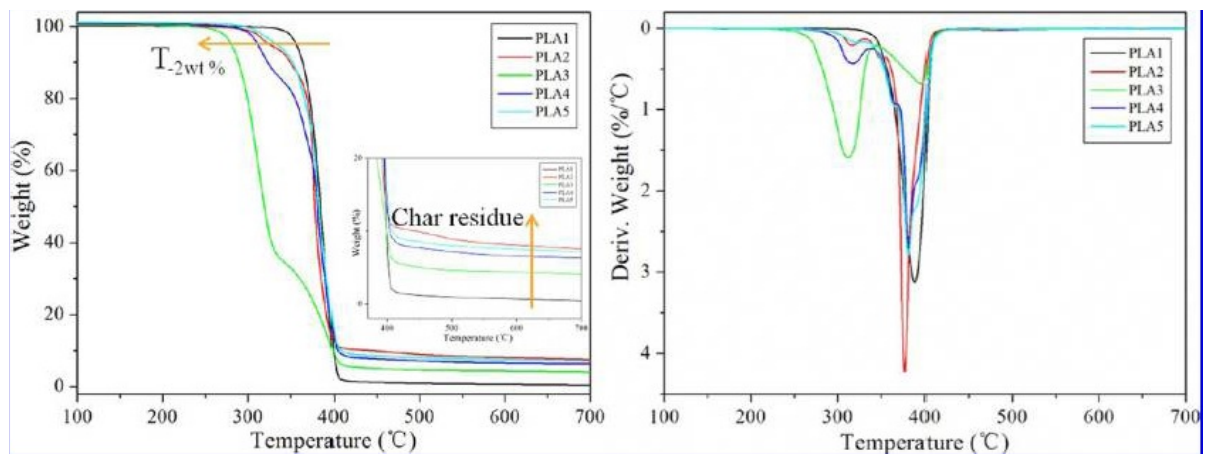

Figure 7. The thermal stability of PLA/HPCP/LDH-SDS composites measured by TGA in a $\mathrm{N}_{2}$ atmosphere at $10{ }^{\circ} \mathrm{C} / \mathrm{min}$.

\subsubsection{Layered metal phosphate-Flame retardants}

Generally, the phosphorus can strengthen the char layers or catalyze the char formation while the metal ion has the ability catalyzing charring effects of polymer. Combining those above effects with the layers structures, the layered metal phosphate/polymer nanocomposites usually exhibit great flame retardant efficiency. 
Among the layered metal phosphate, zirconium bis(monohydrogen orthophosphate) monohydrate ( $\alpha-\mathrm{ZrP})$ is a crystalline, layered compound with many notable features, such as ion-exchange properties, high thermal stability and catalytic activity. As a result, the monohydrogen zirconium phosphates are usually employed to fabricate polymer nanocomposites with good mechanical, thermal and flame-retardant properties. Moreover, due to the weak interactions among the layer nanoplatelets, the intercalation or the exfoliation of the layered compounds in polymeric matrix can be easily achieved via the in situ intercalative polymerization, solution or banburying methods. Most of the studies only focused on the preparation of the nanocomposites and enhancing the thermal and mechanical properties of the nanocomposites, in comparison to the pure polymer matrix. In Tai's work, the copolymer nanocomposites with various amounts of $\alpha-\mathrm{ZrP}$ were successfully prepared by in situ radical bulk polymerization. The completely exfoliated nanocomposites were obtained for the sample containing up to $5 \mathrm{wt} \% \alpha-\mathrm{ZrP}$ relative to the monomers. The TGA data, as shown in Figure 8, indicated that the thermal stability and char yields of the virgin copolymer and nanocomposites were significantly enhanced in comparison to pure polystyrene. Furthermore, the thermal stability of the nanocomposites was found to be dependent on the amount of $\alpha$-ZrP.[59] In the MCC tests (Figure 9), the nanocomposites showed a significant reduction in the heat release capacity (HRC) and total heat release (THR) in comparison to both polystyrene and the flame-retarded copolymer without layered compounds. Based on the MCC tests, the flame-retardant mechanism was ascribed to the increase of final char formation and a decreased mass loss rate. Generally, this study provided a potential approach for the development of novel halogen-free flame-retardants.

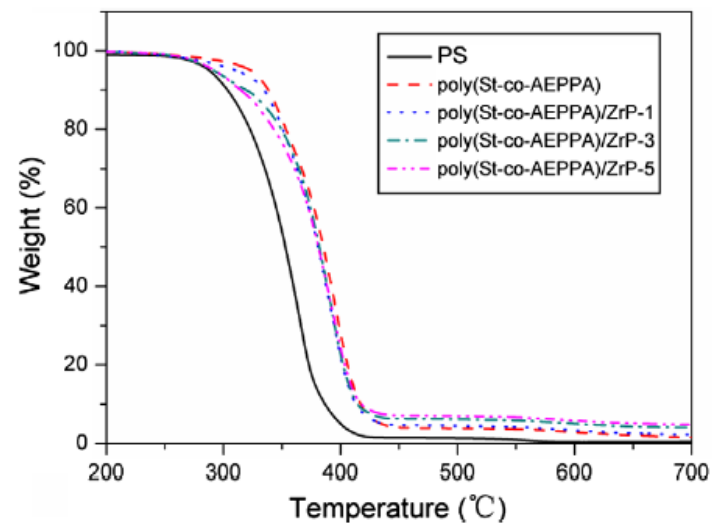

Figure 8. TG curves of (a) PS, (b) poly(St-co-AEPPA), (c) poly(St-co-AEPPA)/ZrP-1, (d) poly(St-co-AEPPA)/ZrP-3, (e) poly(St-co-AEPPA)/ZrP-5 in air atmosphere.

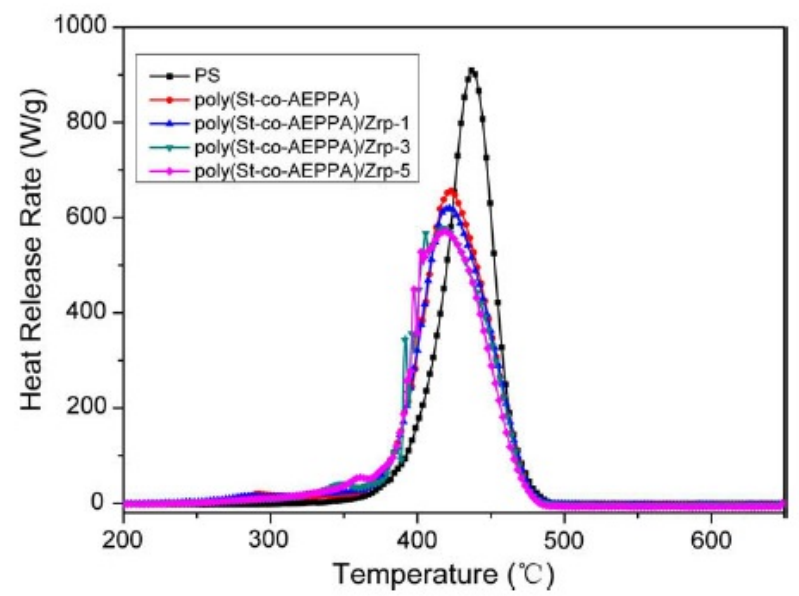

Figure 9. Heat release rate curves of PS and the poly(St-co-AEPPA) nanocomposites in the MCC.

In order to investigate the synergistic effect of the $\alpha-\mathrm{ZrP}$ with conventional flame retardant, Wang prepared PET/ $\alpha-\mathrm{ZrP}$ nanocomposites and the thermal stability as well as the burning behavior of the nanocomposites were investigated.[60] The effect of the small amounts of $\alpha-\mathrm{ZrP}$ on the thermal stability and combustion behaviors are apparent: inhibiting the thermal decomposition and improving the char yields. From the Cone (Figure 10), it was found that the HRR and pHRR were reduced compared to both the pure PET and a fire retarded PET without layered compounds. However, the decrease of THR is not significant. The improved flame retardant properties are related to the formation of the char which could reduce the heat transfer and fuel during the combustion process, and the metal in the nanoparticles could also catalyze the char formation of the polymeric materials. 


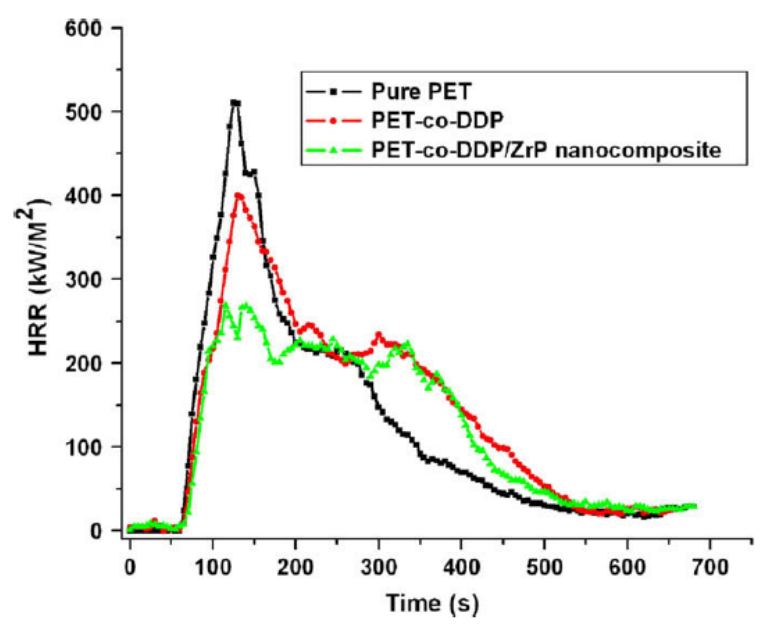

Figure 10. Heat release rate (HRR) for PET, PET/DDP, and PET/DDP/ $\alpha-Z r P$.

In order to further investigated the flame retardant mechanism of the $\alpha-\mathrm{ZrP} /$ polymer nanocomposites. HRTEM was used to further characterize the graphitization of $\alpha-\mathrm{ZrP} / \mathrm{PP}$ residue (Figure 11). Figure 11 clearly shows that the charred residue is comprised of graphite sheets. The image indicates that there are several areas (one shown enlarged) where the structure is aligned in true 3-D order such that both the 002 and the 110 planes are visible (Figure 11(b)). The 002 planes appear as black and white bands and are easily resolved. The 110 planes are harder to identify and run perpendicular to the 002 planes. Figure 11(b) clearly displays the presence of the graphite sheets and the SAED pattern reveals the polycrystalline nature of the graphite. Thus, it can be concluded that the $\alpha$-ZrP could catalyzing the char formation during the combustion. [61]
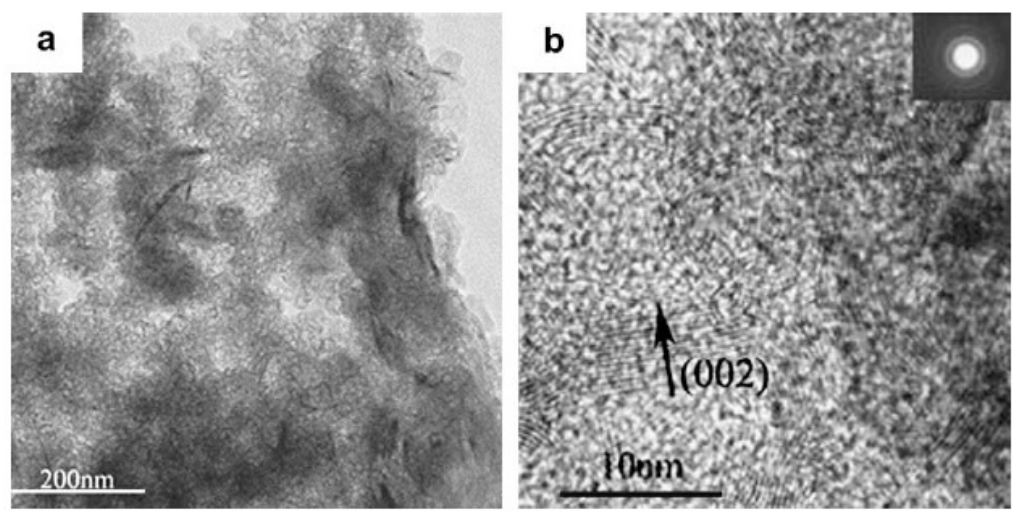

Figure 11. HRTEM images (a) low magnification, (b) high magnification) and SAED pattern of the char residue of PP3

(PP/IFR/2.5 wt\% OZrP nanocomposites).

Similar to the flame retardant effect of $\alpha-\mathrm{ZrP}$, the layered aluminophosphate could also exhibit great flame retardant efficiency. In our previous work, Jiang investigated the flame retardant effects of Poly(methyl methacrylate) (PMMA)/(dodecylamine templated lamellar aluminophosphate) (DDA-LAP) nanocomposites.[62] The results indicated that the nanocomposites kept relatively high transparency in optical property and have a significant improvement in the thermal stability, as shown in Figure 12. The strong interfacial interaction between the aluminophosphate layers and the PMMA chains, the homogeneous dispersion and the formation of graphitized char during heating are considered the three key roles for the properties improvement.

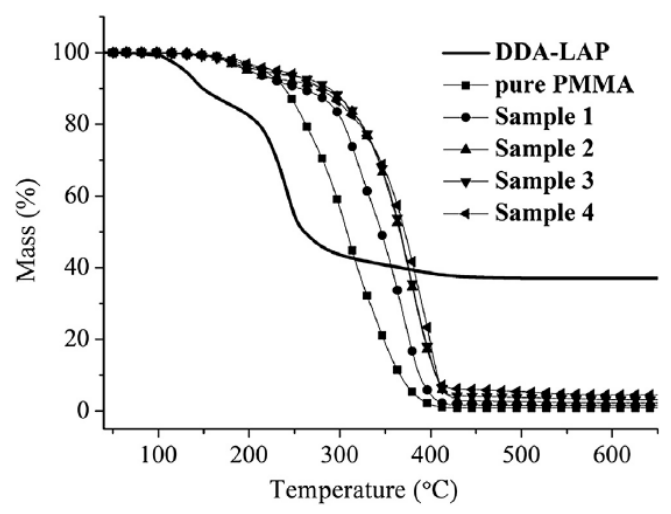

Figure 12. The TGA curves of DDA-LAP, pure PMMA, samples 1-4 in air atmosphere. 
Generally, $\alpha$ - titanium phosphate $(\alpha-\mathrm{TiP})$ is known as an inorganic ion exchangers with a fairly large cation-exchange capacity and the hydroxyl groups in hydrophosphate are attached to the surfaces of $\alpha$-TiP nanolayers, which make $\alpha$-TiP naturally compatible in aqueous media and PVA matrix.[63] Thus, in order to obtain high-performance properties of PVA, $\alpha$-TiP was incorporated into PVA using a facile synthesis strategy based on hydrothermal synthesis, ultrasonic solvent blending, and casting, as shown in Figure 13. The fire resistance was significantly improved with sharply reduced maximum decomposition value (60\%), pHRR (79\%), and THR (25\%), as shown in Figure13 (d). As a conclusion, the strategy for preparing high performance PVA nanocomposites is facile and efficient, indicating the promising use in academic research and practical applications.
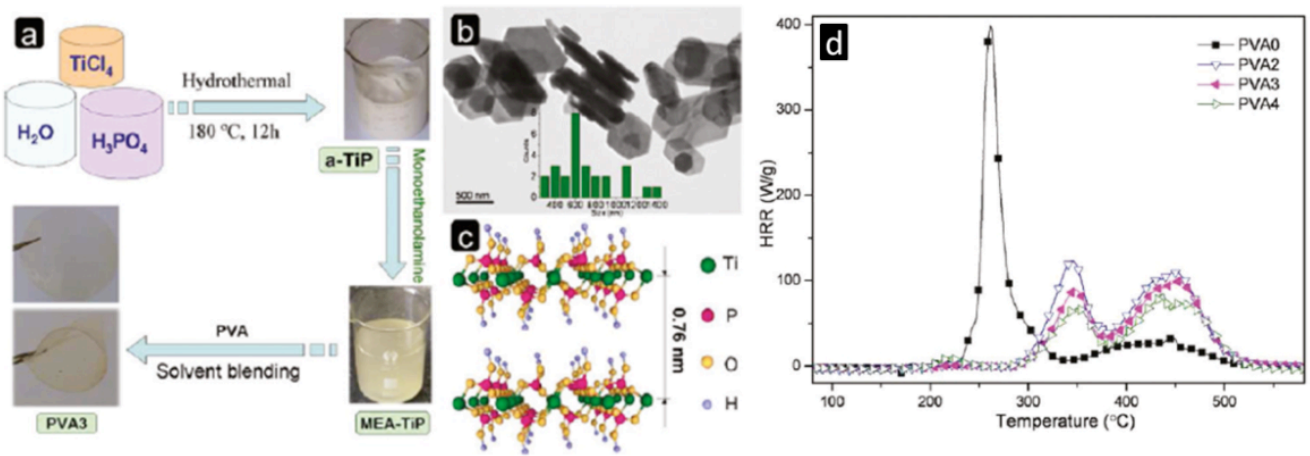

Figure 13. (a) Preparation route of PVA/ $\alpha$-TiP nanocomposite, (b) TEM image of $\alpha$-TiP, and (c) sketch of $\alpha$-TiP structure. The green statistical histogram in panel $b$ shows the sizes of the $\alpha$-TiP particles. (d) Heat release curves obtained from MCC.

Similar to MMT, the use of layered metal phosphate could impart improved flame retardancy for some polymers or flame retardant systems. Although the physical and catalyzing charring effects of layered metal phosphate are believe to be responsible for the enhanced properties, more evidences should be further explored. For example, how do the layered metal phosphate nanoplatelets accelerate the carbonization? What is the mechanism of the catalytic fire retardancy process?

\subsubsection{Graphene based-Flame retardants}

The nanocomposites with improved flame retardancy at low layered compounds loading are highly attractive for industry and academia. In addition to clay, carbon nano-additives such as GO, graphene or expandable graphite have been widely investigated to enhance the flame retardant performance of polymeric materials. Similar to other nanocomposites, there are three main methods of preparing the graphene based nanocomposites: (1) the melt blending, (2) the solvent blending, and (3) the in situ polymerization. Due to the strong interactions among the graphene nanolayers, graphene aggregates easily in the polymer matrix, especially when the graphene are dried. As a result, the strong tendency to re-aggregation makes it difficult for the graphene to disperse well in the polymer matrix by only melt blending. As compared with solvent blending, the in situ polymerization is obviously advantageous in the interface interaction when the monomer is copolymerized with the graphene.

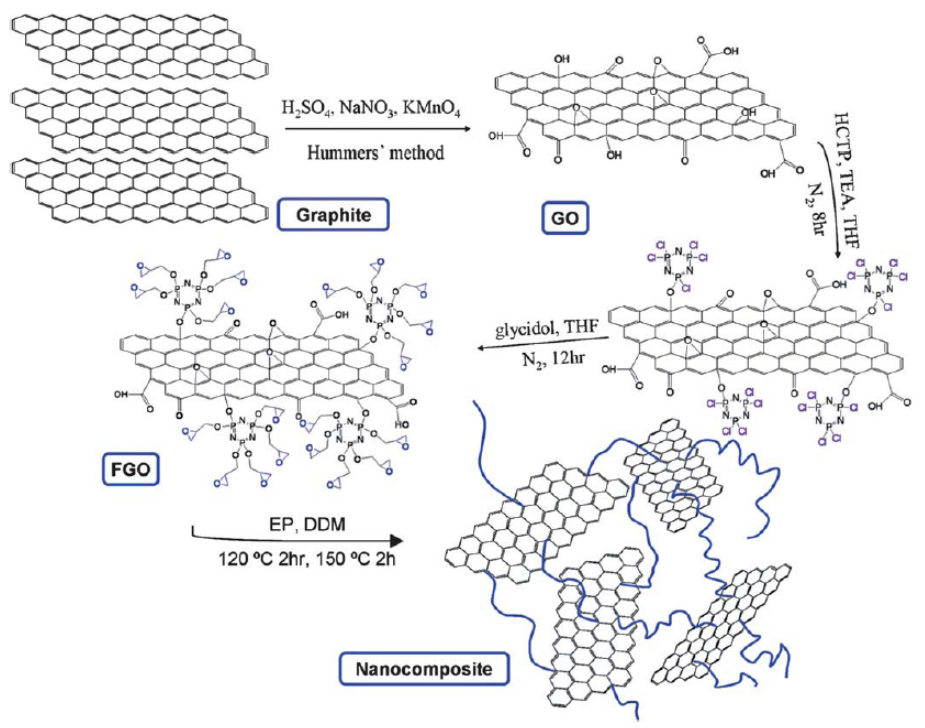

Figure 14. Preparation route $\mathrm{FGO}$ and FGO/EP nanocomposites.

Graphene or GO, as the layer materials, are typical two-dimensional layered inorganic material composed of layered graphite sheets which possess functional reactive groups. These functional groups enable GO or graphene to absorb polar molecules and polar polymers easily to form polymer/layered compounds nanocomposites. Polymer/GO or polymer/graphene nanocomposites 
prepared by in situ polymerization usually had higher thermal stability and increased char formation compared to pure polymer. Bao et al. reported flame-retardant functionalization method to introduce epoxy groups on the graphene surface using hexachlorocyclotriphosphazene (HCTP) as a modifier, as shown in Figure 14.[64] The functionalized graphene was in situ heat-cured with epoxy resin and good dispersion of the graphene was obtained, as shown in Figure 15. The storage modulus, hardness, electrical conductivity and thermal stability of the nanocomposites were improved greatly, which are due to the homogeneous dispersion of graphene and the effect of the flame retardant elements.
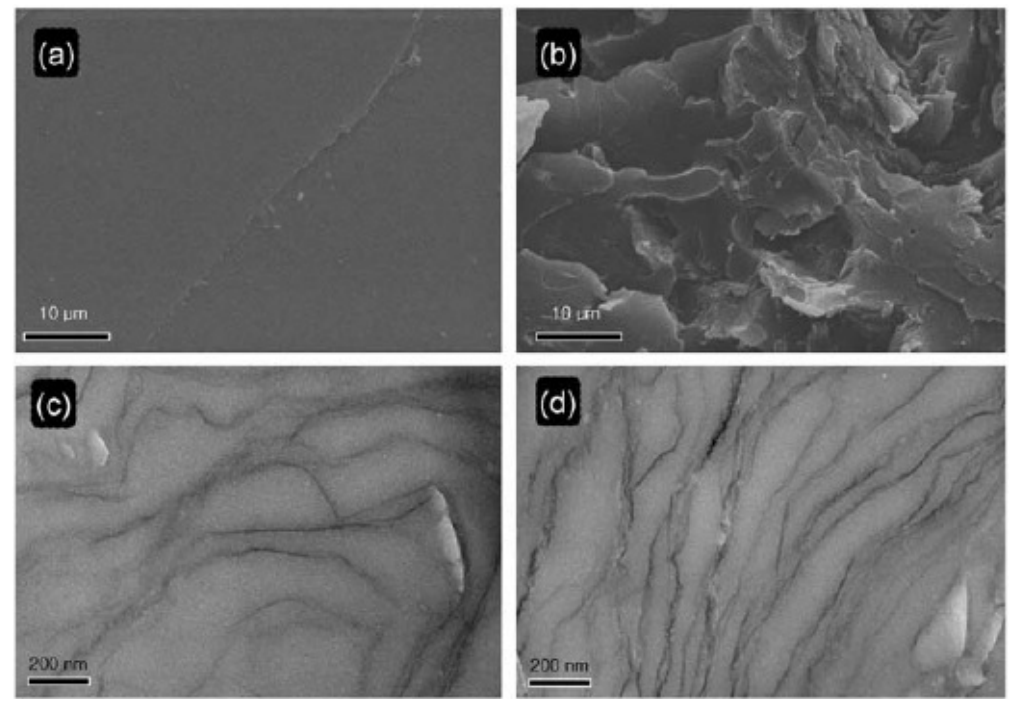

Figure 15. Electron microscopy images of EP and nanocomposites: (a) SEM observation of the fracture surface of EP, (b) SEM micrograph of the fracture surface of EP/1\%FGO nanocomposites, (c) TEM observation of the ultrathin section of EP/1\%FGO nanocomposites, (d) TEM observation of the ultrathin section of EP/2\%FGO nanocomposites

As for the flame retardancy of the graphene-based materials, although the phosphorus and nitrogen-functionalized graphene has been prepared and incorporated into epoxy resins by Bao, the flame retardant efficiency is not high because the flame retardant elements such as phosphorus in the nanocomposites could not reach the high loading level. Thus, improving the loading of phosphorus in the nanocomposites is the challenge that we face with. Compared with phosphorus and nitrogen containing flame retardants, the organic/inorganic flame retardants, which contain both silicon and phosphorus, are promising flame retardants. Silicon usually plays its role in the condensed phase as char reinforcer, whereas phosphates, such as DOPO, play their role in the condensed phase as carbonizing agent or in the gas phase as radical traps. Thus, Qian prepared flame retardant containing graphene, which could facilitate the dispersion of the graphene in the polymer matrix and improve the loading of flame retardant elements in the polymeric matrix, as shown in Figure 16. [65] The pre-exfoliation of graphene in the flame retardants ensures the homodisperse of graphene in the epoxy matrix. Moreover, the combustion of the FRs-rGO/EP nanocomposites has also been reduced, especially the pHRR (Figure 17), indicating that FRs-rGO/EP is highly effective in reducing the fire hazards of EP. The nanocomposites can reach the UL-94 V-0 rate when 5wt.\% FRs-rGO was incorporated into the EP matrix, which are due to the appropriate loading of the flame retardant elements. This flame retardant strategy of FRs-rGO hybrids combines condensed phase and gas phase flame retardant strategies such as the nanocomposites technique, phosphorus-silicon synergism systems in the condensed phase and DOPO flame retardant systems in the gas phase, thus resulting in significant improvements in the flame retardancy of epoxy resins.
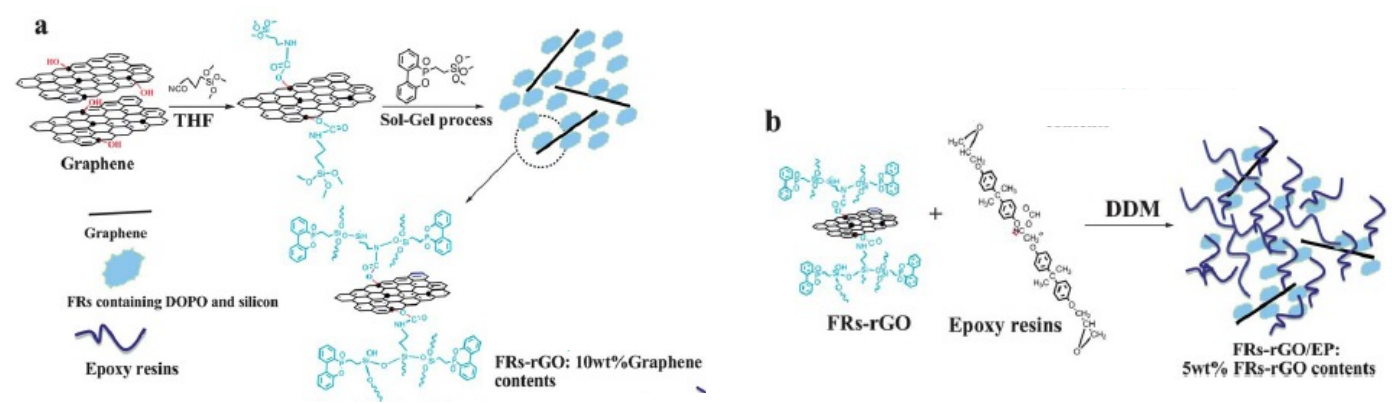

Figure 16. Preparation of FRs-rGO hybrids and FRs-rGO/EP nanocomposites: (a) preparation routes of FRs-rGO hybrids, (b) preparation routes of FRs-rGO/EP nanocomposites. 

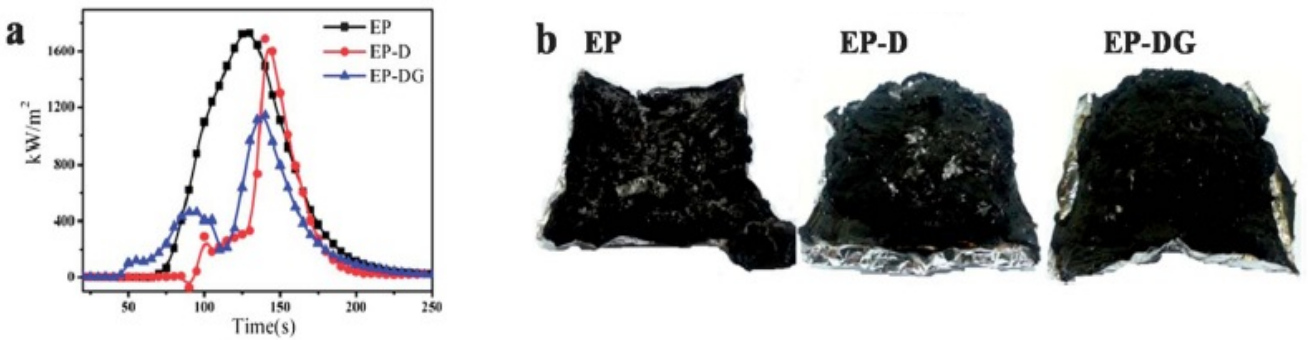

Figure 17. (a) Cone calorimeter test results: HRR curves and (b) digital photographs of char residues after cone test.

Generally, the modification of the GO is usually based on the organic groups and the modification process is uncontrollable. Considering the size-controllability of silicon nanoparticles through the sol-gel process, the decoration of graphene with silicon nanoparticles, which could vary the aspect ratio of graphene through altering the size of silicon nanoparticles, was proposed. In our previous work, the graphene was decorated with organic/inorganic nanoparticles through an in situ sol-gel process with various thicknesses, as shown in Figure 18. [66] The thickness of the modified graphene could be varied by adjusting the amount of the silane additive (Figure 19). Compared with the reduced GO (rGO), the nanoparticles could significantly improve the thermal stability and thermal conductivity of polyurea, implying that the good dispersion of rGO and the functional groups on the surface of rGO had a significant effect on the thermal stability and thermal conductivity of polyurea. Moreover, the peak heat release rate (pHRR) of nanoparticles-rGO/polyurea nanocomposites was significantly reduced, which indicated that the combustible gas releasing rate of polyurea was reduced.

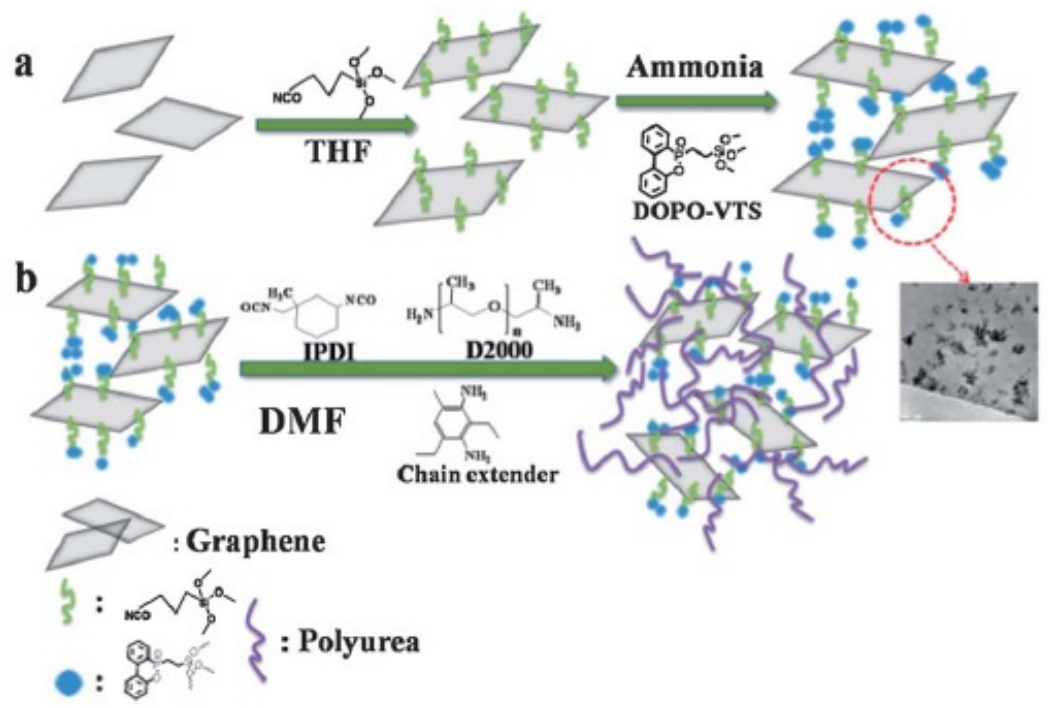

Figure 18. Preparation routes of (a) nanoparticles-rGO and (b) nanoparticles-rGO/polyurea nanocomposites

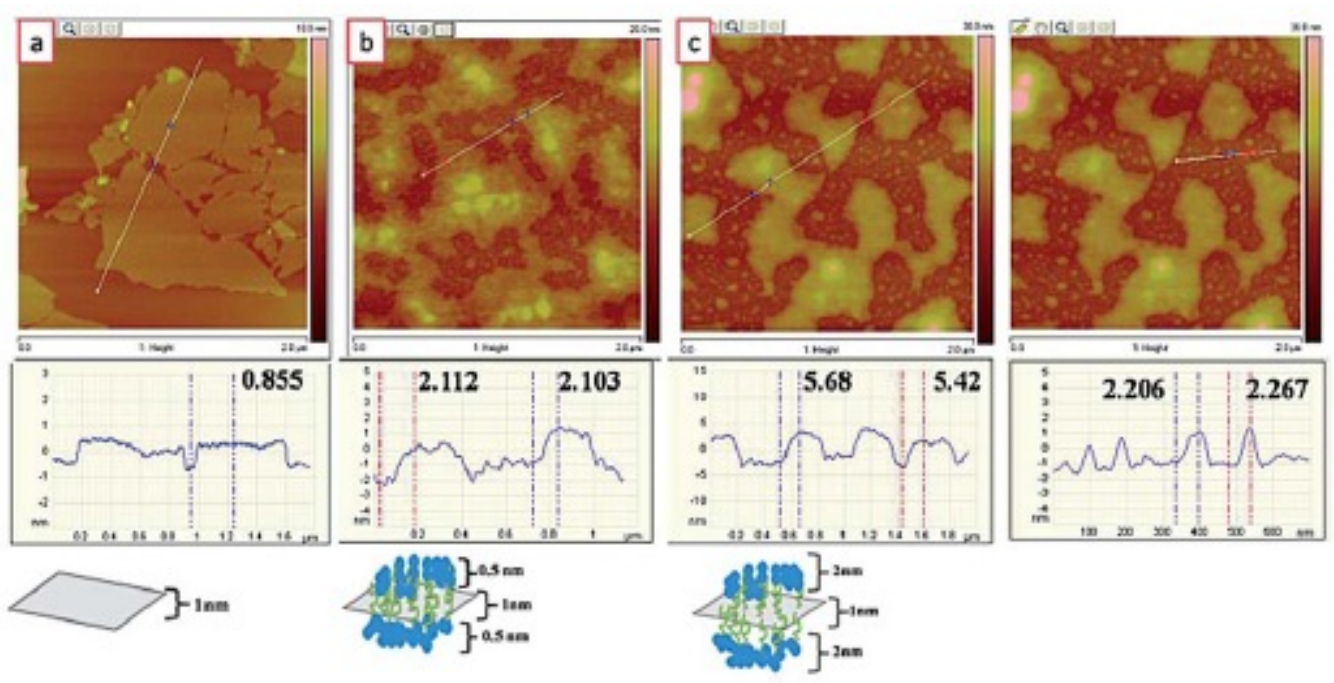

Figure 19. Atomic Force Microscope (AFM) images of (a) GO, (b) nanoparticles-rGO-1 and (c) nanoparticles-rGO-2.

In addition to the flame retardant efficiency of the nanocomposites, the release of smoke and toxic gases in fires are another cause of injury and death. The discovery in our previous work convinced that the amount of heat and toxic gas released are 
decreased during the combustion of graphene/polymer nanocomposites as compared with virgin polymers. Moreover, reducing the toxicity of pyrolysis gases has gained increasing attention in safety science and engineering.[67] Thus, in our previous work, $\mathrm{Co}_{3} \mathrm{O}_{4}$ loaded graphene $\left(\mathrm{Co}_{3} \mathrm{O}_{4} /\right.$ graphene $)$ was prepared and incorporated into the polymer matrix. It was found that $\mathrm{Co}_{3} \mathrm{O}_{4}$ /graphene could significantly reduce the release of gaseous products and the heat release rate, attributing to the barrier effect that the grapheme, which could delay the heat and mass transfer between gas and condensed phases. Furthermore, most of the poisonous $\mathrm{CO}$ gas, which was emitted from polyesters during thermal decomposition were oxidized by $\mathrm{Co}_{3} \mathrm{O}_{4} / \mathrm{graphene}$ hybrids, which was in accordance with the cone results, as shown in Figure 20. The combination of graphene and metal-compounds may contribute a promising strategy to the improvement of the fire safety of polymers.
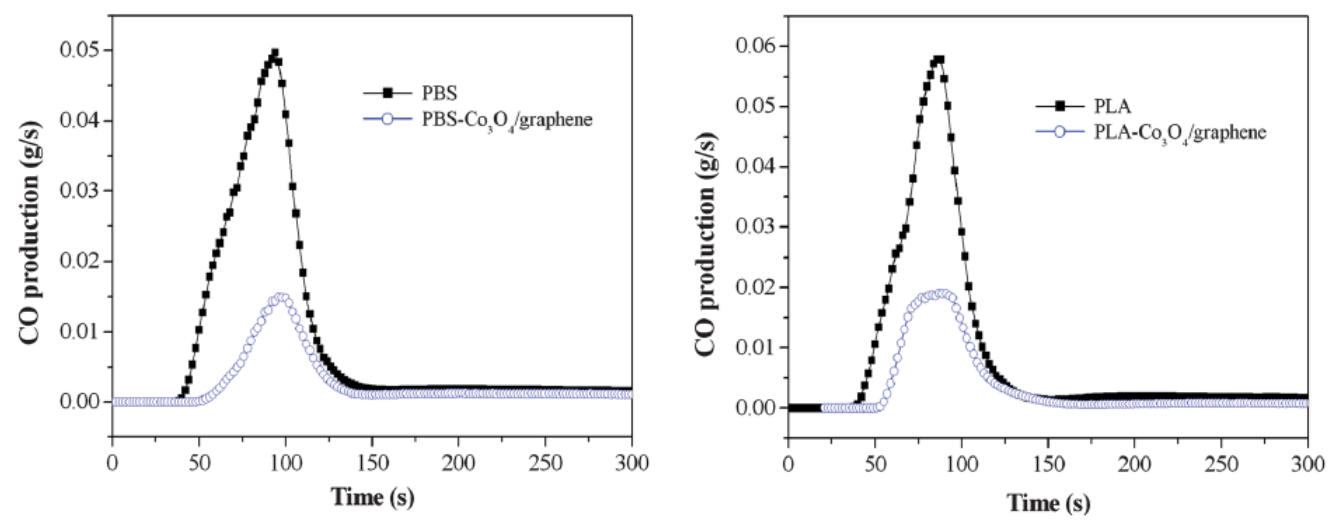

Figure 20. The $\mathrm{CO}$ production curves of PBS, PLA and their $\mathrm{Co}_{3} \mathrm{O}_{4} /$ graphene composites.

Generally, the graphene can be modified with the organic compounds, be decorated with metallic oxide or dispersed in the flame retardants. Commonly, the graphene could generally play the roles of shielding effect and the modifiers could improve the flame retardancy or inhibit the smoke formation. Moreover, the flame-retardant/graphene hybrids could impart the polymer with high flame retardant efficiency due to the appropriate loading of flame retardant elements.

\section{The flame retardant mechanism}

This section is mainly focus on the mechanism of the layered compounds on the reduction of the heat and toxic gases, which lays mainly on the barrier effect and the catalytic charring effect. It appeared that the flame retardant mechanism for polymer/layered compounds nanocomposites was the formation of a continuous protective layer, which appeared to act as heat shield. Moreover, the metal ions or the flame retardant elements could also catalyze the formation of char layers. Thus, it was believed that the barrier effect of the exfoliated layered materials on volatiles played an important role in delaying the thermal-oxidative degradation and the decreasing the pHRR. Compared to pure polymer, the mass loss rate for polymer/layered compound composites are usually slowed down, which are due to the good dispersion of the layered compounds.

The previous works showed that the pHRR could be reduced significantly using the layer materials when the layered materials were well dispersed in polymer matrixes. [53] Although the pHRR was significantly decreased in the previous works, the decreases on THR were not usually significantly. Those could be explained as follows: the burning rate or fire size of these nanocomposites could be slowed by the incorporation of the layered compounds. However, the nanocomposites would usually burn out completely and the polymer would consume the same amount of oxygen, which result in almost the same THR. As for the effect of the char residues, this can be explained as follows: since the boiling point of the most volatiles is much lower than the decomposition temperature of polymer matrix, the volatiles are superheated as they are generated. Then the bubbles nucleate and grow below the heated polymer surface and are released into the gases phase. These bubbles agitate the melt polymer surface and can interfere with the formation of carbonaceous char layer and heat transfer barrier at the polymer surface. The reduced flammability of polymer/layered compounds nanocomposites can be best explained by enhanced char formation in the condensed phase. However, the presence of layered compounds in the polymer matrix retard the vigorous bubbling process and facilitate the formation of a continuous protective char layer at the polymer surface. The layered compounds on the burning surface can insulate the underlying polymeric substrate and thus slow the heat and mass transfer between gaseous and condensed phase. [68] The phenomenon during the thermal degradation of polymer/clay nanocomposites were investigated and confirmed by Kashiwagi (Figure 21). 

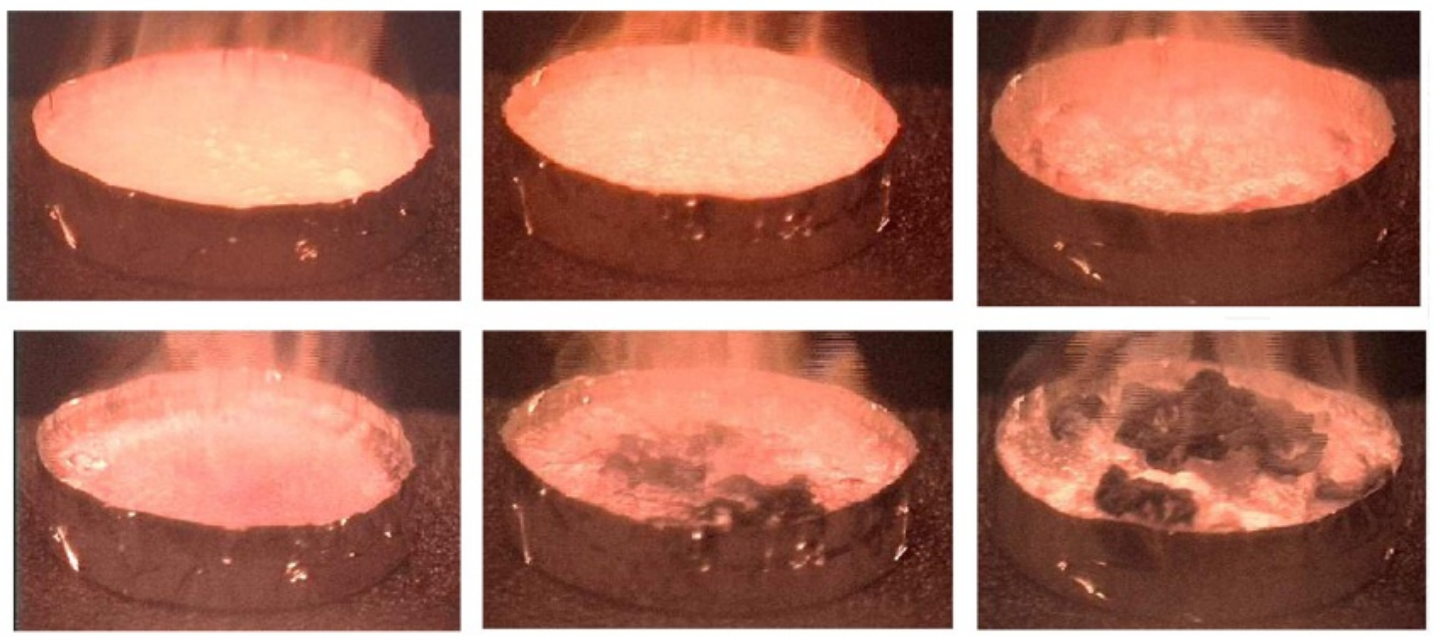

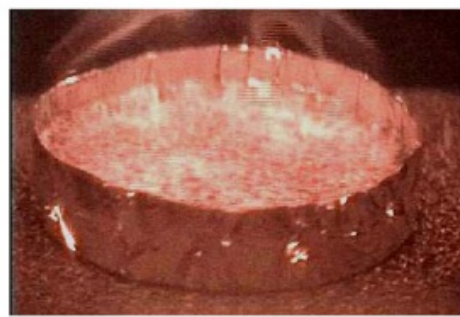

PA6

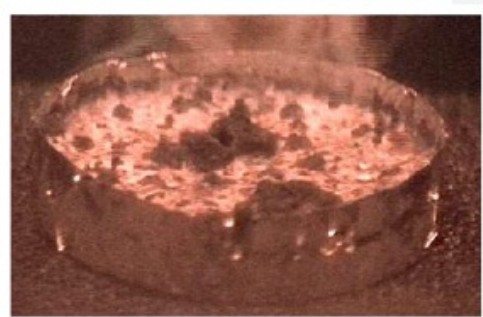

PA6/Clay $(2 \%)$

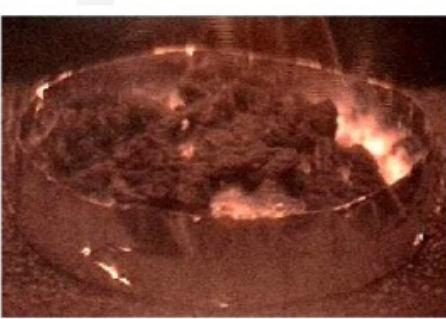

PA6/Clay(5\%)

Figure 21. Selected video images at 100,200 , and $400 \mathrm{~s}$ in nitrogen at $50 \mathrm{~kW} / \mathrm{m}^{2}$.

The barrier effect of the layered clay could improve the flame retardancy of polymer, thus, the transformation of clay during the combustion may also play the flame retardant roles under thermal radiation. Thus, Tan investigated the transformation of clay under heat at the present of different concentrations of air (Figure 22). The results indicate that the clays migrate to the surface of the nanocomposites and the migration of exfoliated platelets to the surface of molten nanocomposites hinders the penetration and diffusion of oxygen into the melt. [69] The migration usually creates a barrier that slows down further oxidation, which indicates the possibility of increasing the aging and storage stability of nanocomposites and increasing their shelf life. Thus, the barrier effect and the migration effect of clay could improve the flam retardancy of polymer greatly.
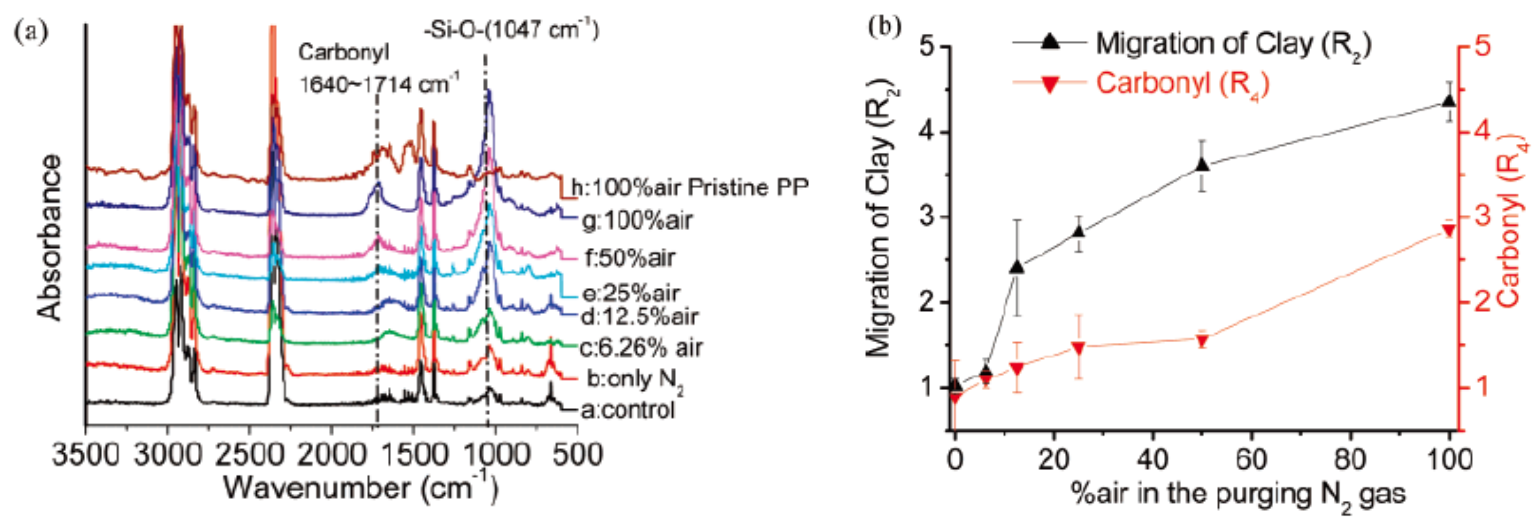

Figure 22. Extent of migration and carbonyl content of samples annealed at $190{ }^{\circ} \mathrm{C}$ under different concentrations of air for 60 min: (a) ATR spectra, (b) R2 and R4 vs \% air.

As for the roles of graphene in the condensed phase, Bao investigated the behaviors of graphene in the condensed phase during the combustion of the polystyrene. After be burning, neat polystyrene does not form any char while the FGO/PS nanocomposites form visible char and the char is increased when the FGO level is increased, as shown in Figure 23. [70] Besides, the inner structure of the char of $\mathrm{PS} / 3 \%$ FGO is bumpy and porous and many layers are observed which imply that FGO is not burned out during the combustion. Many layered structures are visible which could be attributed to the protective FGO layers. The FGO layers and the continuous and compact char surface are good barriers to protect the underlying polymers and inhibit the exchange of degradation products, combustible gases and oxygen. 

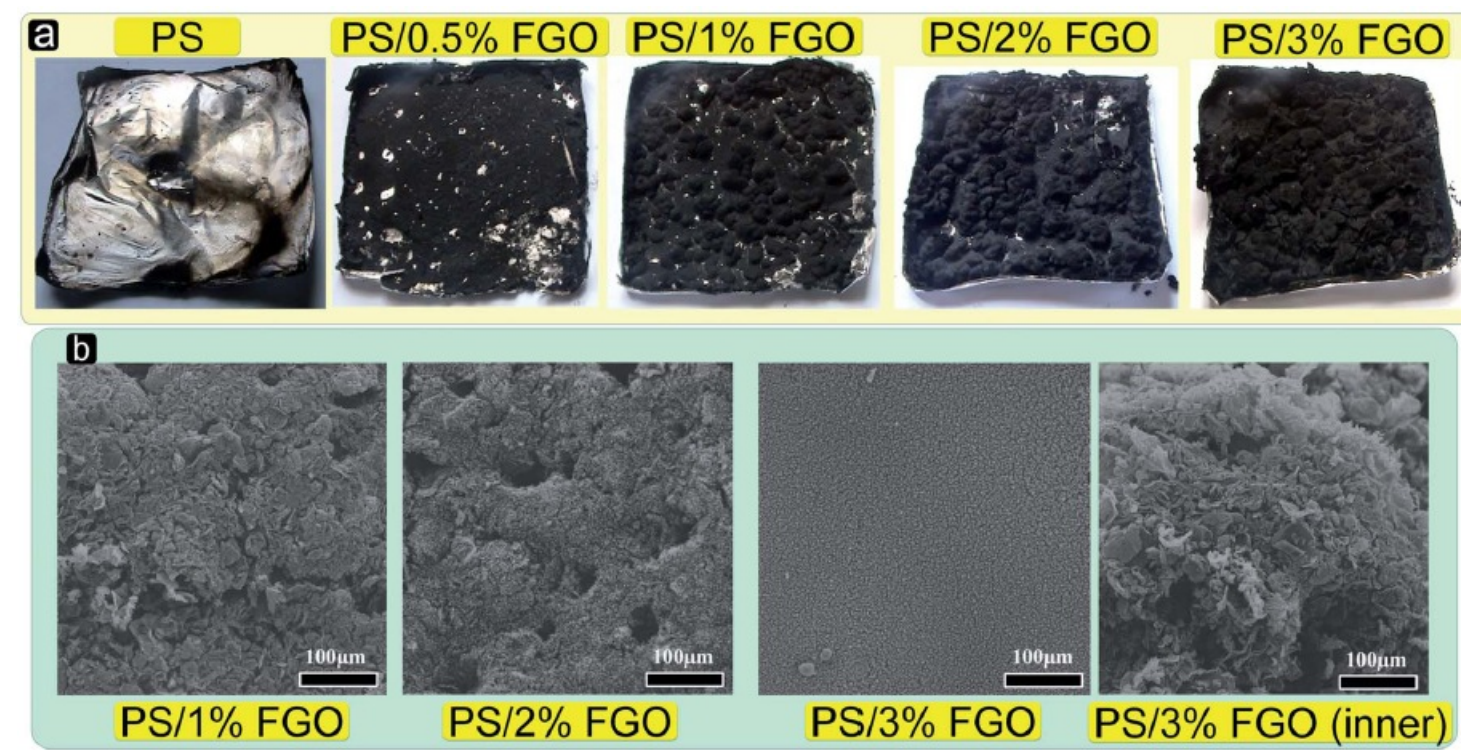

Figure 23. Observations of char: (a) digital photographs and (b) SEM profiles.

Generally, the layered compounds can be modified with the organic compounds or loaded with the metal ions or dispersed in the flame retardants. The layered compounds could play the roles of barrier effect, the modifier could play the role of flame retardancy or the smoke suppression and the silicon based layered compounds could migrate to the surface of the nanocomposites. Moreover, the flame-retardants/layered compounds hybrids could impart the polymer with high flame retardant efficiency due to the appropriate loading of flame retardant elements and the "synergistic effect". The good performances of the layered compounds in the polymeric matrix are due to the following facts: the barrier effect of layered structure, the migration effect and the catalyzing charring effect of the metal ion.

\section{Summary and outlook}

The influence of layered compounds, including MMT, LDH, layered metal phosphate and graphene, on flammability performance of polymer/layered compounds nanocomposites (PLCNs) were reviewed. PLCNs exhibited superior flame retardancy compared to the corresponding pure polymers, due to that layered compounds possess barrier effect. However, the addition of layered compounds could not decrease the total heat release (THR) of PLCNs efficiently; they may be useful as a component of flame retardant systems but not an efficient flame retardant when used alone. Combinations of PLCNs with metal ions and traditional flame retardants are predicted to have high probability to enhance the fire safety of polymeric materials, since they can catalyze the charring process during combustion and restrain the release of combustible gases. However, there is still a long way to go to reach a full understanding and practical principle of the PLCNs.

However, the THR of the nanocomposites was always unchanged when the layered compounds are used alone. Thus, it is unlikely that nanocomposites will ever be used as a stand-alone flame retardant system, but the layered compounds usually possess the barrier effect while the metal ions or the phosphorus play the role of catalyzing charring effect during combustion. The polymer/layered compounds nanocomposites exhibited much lower peak heat release rate (pHRR) compared with pure polymer materials. The layered compounds such as MMT, LDH, layered metal phosphate and graphene can improve the flame retardancy of the polymer matrix and these nanocomposites exhibit better flame retardancy when the layered compounds are combined with traditional flame retardants.

\section{Reference}

1. Lu SY, Hamerton I. (2002) Recent developments in the chemistry of halogen-free flame retardant polymers. Progress in polymer science; 27(8):1661-1712.

2. Kashiwagi T, Du F, Douglas JF, Winey KI, Harris RH, Jr., Shields JR. Nanoparticle networks reduce the flammability of polymer nanocomposites. Nature materials 2005; 4(12): 928-33.

3. Zhang S, Horrocks AR. A review of flame retardant polypropylene fibres. Progress in Polymer Science 2003; 28(11): 1517-38.

4. Qian X, Song L, Jiang S, et al. Novel Flame Retardants Containing 9,10-Dihydro-9-oxa-10-phosphaphenanthrene-10-oxide and Unsaturated Bonds: Synthesis, Characterization, and Application in the Flame Retardancy of Epoxy Acrylates. Industrial \& Engineering Chemistry Research 2013; 52(22): 7307-15.

5. Borreguero AM, Sharma P, Spiteri C, et al. A novel click-chemistry approach to flame retardant polyurethanes. Reactive and 
Functional Polymers 2013; 73(9): 1207-12.

6. Qian X, Song L, Wang B, Hu Y, Yuen RKK. Synthesis of organophosphorus modified nanoparticles and their reinforcements on the fire safety and mechanical properties of polyurea. Materials Chemistry and Physics 2013; 139(2-3): 443-9.

7. Wang X, Xing W, Song L, et al. Flame retardancy and thermal properties of novel UV-curing epoxy acrylate coatings modified by phosphorus-containing hyperbranched macromonomer. Journal of Polymer Research 2013; 20(7).

8. Birnbaum LS, Staskal DF. Brominated Flame Retardants: Cause for Concern? Environmental Health Perspectives 2003; 112(1): 9-17.

9. de Wit, CA .(2002) An overview of brominated flame retardantsin the environment. Chemosphere;46(5):583-624.

10. Alaee M. An overview of commercially used brominated flame retardants, their applications, their use patterns in different countries/regions and possible modes of release. Environment International 2003; 29(6): 683-9.

11. Ferrari M. Cancer nanotechnology: opportunities and challenges. Nature reviews Cancer 2005; 5(3): $161-71$.

12. Paul DR, Robeson LM. Polymer nanotechnology: Nanocomposites. Polymer 2008; 49(15): 3187-204.

13. Hamley IW. Nanotechnologie mit weichen Materialien. Angewandte Chemie 2003; 115(15): 1730-52.

14. Gilman JW, Jackson CL, Morgan AB. Flammability Properties of Polymer - Layered-Silicate

Nanocomposites. Polypropylene and Polystyrene. Chemistry of Materials;12(7):1866-1873.

15. Bharadwaj RK. Modeling the Barrier Properties of Polymer-Layered Silicate Nanocomposites. Macromoecules; 34 (26) :9189-9192.

16. Li Y, Tang L, Li J. Preparation and electrochemical performance for methanol oxidation of pt/graphene nanocomposites. Electrochemistry Communications 2009; 11(4): 846-9.

17. Leroux F, Besse JP.Polymer Interleaved Layered Double Hydroxide: A New Emerging Class of Nanocomposites. Chemistry of Materials;13(10):3507-3515.

18. Kashiwagi T, Grulke E, Hilding J, et al. Thermal and flammability properties of polypropylene/carbon nanotube nanocomposites. Polymer 2004; 45(12): 4227-39.

19. Ji B, Gao H. Mechanical properties of nanostructure of biological materials. Journal of the Mechanics and Physics of Solids 2004; 52(9): 1963-90.

20. Jang BN, Wilkie CA. The effect of clay on the thermal degradation of polyamide 6 in polyamide 6/clay nanocomposites. Polymer 2005; 46(10): 3264-74.

21. Kashiwagi T, Grulke E, Hilding J, Harris R, Awad W, (2011)Douglas J. Macromolecular Rapid Communications; 48(1):21-30.

22. Zanetti M, Kashiwagi T, Falqui L. Cone Calorimeter Combustion and Gasification Studiesof Polymer Layered Silicate Nanocomposites. Chemistry of Materials; 14(2):881-887.

23. Fina A, Abbenhuis HCL, Tabuani D, Camino G. Metal functionalized POSS as fire retardants in polypropylene. Polymer Degradation and Stability 2006; 91(10): 2275-81.

24. Kawasumi, M; Hasegawa, N; Kato, M. Preparation and Mechanical Properties of Polypropylene - Clay Hybrids. Macromolecules; 30(20):6333-6338.

25. Zanetti, M; Kashiwagi, T; Falqui, L. Cone Calorimeter Combustion and Gasification Studies of Polymer Layered Silicate Nanocomposites. Chemistry of Materials; 14(2):881-887.

26. Sinha Ray S, Okamoto M. Polymer/layered silicate nanocomposites: a review from preparation to processing. Progress in Polymer Science 2003; 28(11): 1539-641.

27. Paul DR, Robeson LM. Polymer nanotechnology: Nanocomposites. Polymer 2008; 49(15): 3187-204.

28. Pavlidou S, Papaspyrides CD. A review on polymer-layered silicate nanocomposites. Progress in Polymer Science 2008; 33(12): 1119-98.

29. Alexandre, M; Dubois, P. Polymer-layered silicate nanocomposites: preparation, properties and uses of a new class of materials. Materials Science \& Engineering-reports; 28(1-2):1-63.

30. Choy JH; Kwak, SY; Jeong, YJ. inorganic layered double hydroxides as nonviral vectors. Angewandte Chemie-Internationa Edition;39(22):4042-4045.

31. Newman, SP; Jones, W. Synthesis, characterization and applications of layered double hydroxides containing organic guests. New Journal Of Chemistry;22(6):649-649.

32. Khan, AI; O, Hare D. Intercalation chemistry of layered double hydroxides: recent developments and applicationsBasis of a 
presentation given at Materials Discussion No. 5, 22-25 September 2002, Madrid, Spain. Journal of Materials Chemistry 2002; 12(11): 3191-8.

33. Choudary, BM; Madhi, S; Chowdari, NS . Layered Double Hydroxide Supported Nanopalladium Catalyst for Heck-, Suzuki-, Sonogashira-, and Stille-Type Coupling Reactions of Chloroarenes. Journal Of The American Chemical Society;124(47): 14127-14136.

34. Lu H, Wilkie CA. Study on intumescent flame retarded polystyrene composites with improved flame retardancy. Polymer Degradation and Stability 2010; 95(12): 2388-95.

35. Lu H, Wilkie CA, Ding M, Song L. Thermal properties and flammability performance of poly (vinyl alcohol)/ $\alpha$-zirconium phosphate nanocomposites. Polymer Degradation and Stability 2011; 96(5): 885-91.

36. Carosio F, Alongi J, Malucelli G. $\alpha$-Zirconium phosphate-based nanoarchitectures on polyester fabrics through layer-by-layer assembly. Journal of Materials Chemistry 2011; 21(28): 10370.

37. Zheng W; Wong SC. Electrical conductivity and dielectric properties of PMMA/expanded graphite composites. Composites Science and Technology; 63(2):225-235.

38. Yasmin A, Luo J-J, Daniel IM. Processing of expanded graphite reinforced polymer nanocomposites. Composites Science and Technology 2006; 66(9): 1182-9.

39. Stankovich S, Dikin DA, Piner RD, et al. Synthesis of graphene-based nanosheets via chemical reduction of exfoliated graphite oxide. Carbon 2007; 45(7): 1558-65.

40. Dikin DA, Stankovich S, Zimney EJ, et al. Preparation and characterization of graphene oxide paper. Nature 2007; 448(7152): 457-60.

41. Lerf, A; He, HY; Forster, M . Structure of Graphite Oxide Revisited. Journal Of Physical Chemistry;102(23):4477-4482.

42. Zhu Y, Murali S, Cai W, et al. Graphene and graphene oxide: synthesis, properties, and applications. Advanced materials 2010; 22(35): 3906-24.

43. Ferrari AC, Meyer JC, Scardaci V, et al. Raman Spectrum of Graphene and Graphene Layers. Physical Review Letters 2006; 97(18).

44. Geim, A. K. Graphene: Status and Prospects. Science;324(5934):1560-1534.

45. Kashiwagi T, Grulke E, Hilding J, et al. Thermal and flammability properties of polypropylene/carbon nanotube nanocomposites. Polymer 2004; 45(12): 4227-39.

46. Huang G, Gao J, Wang X, Liang H, Ge C. How can graphene reduce the flammability of polymer nanocomposites? Materials Letters 2012; 66(1): 187-9.

47. Guo Y, Bao C, Song L, Yuan B, Hu Y. In Situ Polymerization of Graphene, Graphite Oxide, and Functionalized Graphite Oxide into Epoxy Resin and Comparison Study of On-the-Flame Behavior. Industrial \& Engineering Chemistry Research 2011; 50(13): 7772-83.

48. Tai Q, Yuen RKK, Song L, Hu Y. A novel polymeric flame retardant and exfoliated clay nanocomposites: Preparation and properties. Chemical Engineering Journal 2012; 183: 542-9.

49. Wang B, Song L, Hong N, Tai Q, Lu H, Hu Y. Effect of electron beam irradiation on the mechanical and thermal properties of intumescent flame retarded ethylene-vinyl acetate copolymer/organically modified montmorillonite nanocomposites. Radiation Physics and Chemistry 2011; 80(11): 1275-81.

50. Kong Q, Tang Y, Hu Y, Song L, Liu H, Li L. Thermal stability and flame retardance properties of acrylonitrile-butadiene-styrene/polyvinyl chloride/organophilic Fe-montmorillonite nanocomposites. Journal of Polymer Research $2011 ; 19(1)$.

51. Cai Y, Wu N, Wei Q, et al. Structure, surface morphology, thermal and flammability characterizations of polyamide6/organic-modified Fe-montmorillonite nanocomposite fibers functionalized by sputter coating of silicon. Surface and Coatings Technology 2008; 203(3-4): 264-70.

52. Nazaré S, Kandola B K, Horrocks A R. Flame-retardant unsaturated polyester resin incorporating nanoclay. 2006;17(4): 294-303.

53. Dasari A, Yu Z-Z, Mai Y-W, Liu S. Flame retardancy of highly filled polyamide 6/clay nanocomposites. Nanotechnology 2007; 18(44): 445602.

54. Hsueh HB; Chen CY. Preparation and properties of LDHs/polyimide nanocomposites. Polymer;44(4):1151-1161.

55. Qiu L, Chen W, Qu B. Morphology and thermal stabilization mechanism of LLDPE/MMT and LLDPE/LDH 
nanocomposites. Polymer 2006; 47(3): 922-30.

56. Wang G-A, Wang C-C, Chen C-Y. The disorderly exfoliated LDHs/PMMA nanocomposite synthesized by in situ bulk polymerization. Polymer 2005; 46(14): 5065-74.

57. Xing W, Song L, Wang X, Lv X, Hu Y. Preparation, combustion, and thermal behavior of UV-cured epoxy-based coatings containing layered double hydroxide. Polymers for Advanced Technologies 2011; 22(12): 1859-64.

58. Shan X, Song L, Xing W, Hu Y, Lo S. Effect of Nickel-Containing Layered Double Hydroxides and Cyclophosphazene Compound on the Thermal Stability and Flame Retardancy of Poly(lactic acid). Industrial \& Engineering Chemistry Research 2012; 51(40): 13037-45.

59. Tai Q, Kan Y, Chen L, Xing W, Hu Y, Song L. Morphologies and thermal properties of flame-retardant polystyrene/ $\alpha$-zirconium phosphate nanocomposites. Reactive and Functional Polymers 2010; 70(6): 340-5.

60. Wang D-Y, Liu X-Q, Wang J-S, Wang Y-Z, Stec AA, Hull TR. Preparation and characterisation of a novel fire retardant PET/ $\alpha$-zirconium phosphate nanocomposite. Polymer Degradation and Stability 2009; 94(4): 544-9.

61. Yang D-D, Hu Y, Song L, Nie S B, He S-Q, Cai Y-B. Catalyzing carbonization function of a-ZrP based intumescent fire retardant polypropylene nanocomposites. Polymer Degradation and Stability , 2008 ;93(2), 2014 - 2018.

62. Jiang S, Gui Z, Hu Y, Zhou K, Dong Y, Shi Y. The intercalation of poly(methyl methacrylate)/aluminophosphate nanocomposites and the properties improvement. Materials Chemistry and Physics 2013; 141(1): 95-100.

63. Bao C, Guo Y, Song L, Lu H, Yuan B, Hu Y. Facile Synthesis of Poly(vinyl alcohol)/ $\alpha$-Titanium Phosphate Nanocomposite with Markedly Enhanced Properties. Industrial \& Engineering Chemistry Research 2011; 50(19): 11109-16.

64. Bao C, Guo Y, Song L, Kan Y, Qian X, Hu Y. In situ preparation of functionalized graphene oxide/epoxy nanocomposites with effective reinforcements. Journal of Materials Chemistry 2011; 21(35): 13290.

65. Qian X, Song L, Yu B, et al. Novel organic-inorganic flame retardants containing exfoliated graphene: preparation and their performance on the flame retardancy of epoxy resins. Journal of Materials Chemistry A 2013; 1(23): 6822.

66. Qian X, Yu B, Bao C, et al. Silicon nanoparticle decorated graphene composites: preparation and their reinforcement on the fire safety and mechanical properties of polyurea. Journal of Materials Chemistry A 2013; 1(34): 9827.

67. Wang X, Song L, Yang H, Xing W, Lu H, Hu Y. Cobalt oxide/graphene composite for highly efficient CO oxidation and its application in reducing the fire hazards of aliphatic polyesters. Journal of Materials Chemistry 2012; 22(8): 3426.

68. Kashiwagi T, Harris RH, Zhang X, et al. Flame retardant mechanism of polyamide 6-clay nanocomposites. Polymer 2004; 45(3): 881-91.

69. Lewin M, Tang Y. Oxidation-Migration Cycle in Polypropylene-Based Nanocomposites. Macromolecules 2008, 41(4), $13-17$

70. Bao C, Guo Y, Yuan B, Hu Y, Song L. Functionalized graphene oxide for fire safety applications of polymers: a combination of condensed phase flame retardant strategies. Journal of Materials Chemistry 2012; 22(43): 23057. 